\title{
Article
}

\section{Titanium Nitride as a New Prospective Material for NanoSQUIDs and Superconducting Nanobridge Electronics}

\author{
Michael I. Faley ${ }^{1, *(D)}$, Yuchen Liu ${ }^{1,2}$ and Rafal E. Dunin-Borkowski ${ }^{1,3}$ \\ 1 Peter Grünberg Institute 5, Forschungszentrum Jülich GmbH, 52425 Jülich, Germany; \\ yuchen.liu@rwth-aachen.de (Y.L.); r.dunin-borkowski@fz-juelich.de (R.E.D.-B.) \\ 2 Faculty of Georesources and Materials Engineering, RWTH Aachen University, 52062 Aachen, Germany \\ 3 Ernst Ruska-Centre for Microscopy and Spectroscopy with Electrons, Forschungszentrum Jülich GmbH, \\ 52425 Jülich, Germany \\ * Correspondence: m.faley@fz-juelich.de
}

check for updates

Citation: Faley, M.I.; Liu, Y.; Dunin-Borkowski, R.E. Titanium Nitride as a New Prospective Material for NanoSQUIDs and Superconducting Nanobridge Electronics. Nanomaterials 2021, 11, 466. https://doi.org/10.3390/ nano11020466

Academic Editor: Jeong Ryeol Choi Received: 20 January 2021

Accepted: 9 February 2021

Published: 12 February 2021

Publisher's Note: MDPI stays neutral with regard to jurisdictional claims in published maps and institutional affiliations.

Copyright: (c) 2021 by the authors. Licensee MDPI, Basel, Switzerland. This article is an open access article distributed under the terms and conditions of the Creative Commons Attribution (CC BY) license (https:/ / creativecommons.org/licenses/by/ $4.0 /)$.

\begin{abstract}
Nanobridge Josephson junctions and nanometer-scale superconducting quantum interference devices (nanoSQUIDs) based on titanium nitride (TiN) thin films are described. The TiN films have a room temperature resistivity of $\sim 15 \mu \Omega \cdot \mathrm{cm}$, a superconducting transition temperature $T_{\mathcal{C}}$ of up to $5.3 \mathrm{~K}$ and a coherence length $\xi(4.2 \mathrm{~K})$ of $\sim 105 \mathrm{~nm}$. They were deposited using pulsed DC magnetron sputtering from a stoichiometric TiN target onto Si (100) substrates that were heated to $800{ }^{\circ} \mathrm{C}$. Electron beam lithography and highly selective reactive ion etching were used to fabricate nanoSQUIDs with 20-nm-wide nanobridge Josephson junctions of variable thickness. X-ray and high-resolution electron microscopy studies were performed. Non-hysteretic $I(V)$ characteristics of the nanobridges and nanoSQUIDs, as well as peak-to-peak modulations of up to $17 \mu \mathrm{V}$ in the $V(B)$ characteristics of the nanoSQUIDs, were measured at $4.2 \mathrm{~K}$. The technology offers prospects for superconducting electronics based on nanobridge Josephson junctions operating within the framework of the Ginzburg-Landau theory at $4.2 \mathrm{~K}$.
\end{abstract}

Keywords: titanium nitride; nanobridge Josephson junction; nanoSQUID; superconducting electronics

\section{Introduction}

Superconducting electronics exhibit lower energy consumption, higher speed and lower noise than semiconductor electronics. However, the miniaturization of superconducting circuits is challenging. A reduction in the thickness and width of superconducting lines to below $\sim 100 \mathrm{~nm}$ can lead to an increase in their kinetic inductance. Smaller Josephson junctions (JJs) without shunting resistances and higher critical current densities $J_{c}$ are required. High values of $J_{c}$ are needed to ensure a sufficiently large Josephson energy $E_{J}$ for stability against thermal fluctuations, as well as to squeeze magnetic flux bias into nanometer-sized loops of superconducting quantum interference devices (SQUIDs) or cells of rapid single flux quantum (RSFQ) circuits. The use of nanobridge JJs is advantageous for the miniaturization of SQUIDs and other superconducting circuits because they provide the highest possible values of $J_{c}$, which can approach the depairing critical current density, in superconducting films ( $30 \mathrm{MA} / \mathrm{cm}^{2}$ in $\mathrm{Nb}$ thin films).

Nanobridge JJs have negligible capacitance when compared with tunnel JJs. However, they often have hysteretic $I(V)$ characteristics due to overheating or a multivalued current-phase dependence $I(\varphi)$. Thermal hysteresis is observed in nanobridge JJs that have sufficiently large critical currents $I_{\mathcal{C}}$, while the $I(\varphi)$ dependence becomes multivalued when the length $L$ of a nanobridge JJ exceeds the coherence length $\xi$ by a factor of $\sim 3.5$ [1], depending on the shape of the nanobridge. In $\mathrm{Nb}$ thin films, $\xi(0 \mathrm{~K})$ is $\sim 10 \mathrm{~nm}$, resulting in the need for sophisticated $10 \mathrm{~nm}$ preparation technology for non-hysteretic operation [2-4]. Nb-based nanobridge JJs that have a length of $100 \mathrm{~nm}$ show non-hysteretic 
operation only over a narrow temperature range above $7.2 \mathrm{~K}$ [5]. The absence of hysteresis in their $I(V)$ characteristics at higher temperature results from a reduction in $I_{\mathcal{C}}$ of the $\mathrm{JJ}$ in the case of thermal hysteresis and from an increase in $\xi$ as the temperature $T$ of the nanobridge approaches the superconducting transition temperature $T_{\mathcal{C}}$, according to the relation $\xi \propto\left(1-\frac{T}{T_{c}}\right)^{-\frac{1}{2}}$.

It would be more practical to operate nanobridge JJs in hysteresis-free mode at a temperature of $4.2 \mathrm{~K}$, which is naturally stabilized by the boiling of liquid He at atmospheric pressure. A nanobridge length of $\sim 100 \mathrm{~nm}$ can be achieved relatively easily and with adequate reproducibility by using reactive ion etching (RIE). However, $\mathrm{Nb}$ nanobridges of this size are hysteretic at $4.2 \mathrm{~K}$ as a result of their short coherence length. Instead of $\mathrm{Nb}$, a superconducting material that has a value of $T_{\mathcal{C}}$ of $\sim 5 \mathrm{~K}$ and a value of $\xi$ in a thin film of $\sim 50 \mathrm{~nm}$ would be more suitable. Here, we suggest using titanium nitride (TiN) for this purpose, despite the fact that nanobridge JJs were not realized previously from TiN films.

Covalent bonding between $\mathrm{Ti}$ and $\mathrm{N}$ is responsible for the hardness and chemical stability of TiN. It is therefore used to harden surfaces and to protect them against corrosion, as well as for decorative coatings that resemble Au [6]. TiN can be classified as a quantum material as a result of its superconducting and plasmonic properties. Coplanar waveguide resonators based on superconducting TiN films have demonstrated internal quality factors of up to $10^{7}$ [7]. Resonators based on TiN films have much fewer losses than resonators made with $\mathrm{Nb}$, Re or $\mathrm{Al}$ films deposited on the same substrates [8]. The use of superconducting TiN films in shunting capacitors in transmon qubits was found to enhance relaxation and dephasing times six-fold over those obtained using lift-off $\mathrm{Al}$ up to $\sim 60 \mu \mathrm{s}$ [9]. In the latter study, the JJs in the transmon qubits were still Al tunnel JJs made using shadow evaporation, which may have been one of the residual sources of decoherence. It would be important to realize all-TiN qubits to minimize dielectric losses arising from dissipation due to quantum two-level states (TLSs) at oxide interfaces [10], especially in the oxide that forms at the metal-air interface [8].

Epitaxial $8 \times 8 \mu^{2} \mathrm{TiN} / \mathrm{AlN} / \mathrm{TiN}$ tunnel JJs were fabricated on $\mathrm{MgO}$ and characterized [11]. TiN and $\mathrm{MgO}$ have lattice constants of approximately $0.42 \mathrm{~nm}$ and similar rocksalt crystal structures, simplifying epitaxial growth. However, a low critical current density $J_{c}$ of $\sim 50 \mathrm{~A} / \mathrm{cm}^{2}$ and a large subgap leakage current were observed. These features are disadvantageous for applications of tunnel TiN JJs in nanoscale superconducting circuits. The deposition of TiN films on Si substrates would be preferable to minimize the density of TLSs and the oxygen content of TiN, as well as for technological compatibility with other processes, including bulk nanomachining of Si substrates for the fabrication of cantilevers [4]. However, Si has a diamond crystal structure with a lattice constant of $0.54 \mathrm{~nm}$, which corresponds to a $28 \%$ lattice misfit with TiN, complicating epitaxial growth of TiN films on Si. Nevertheless, four-unit cells of TiN match three-unit cells of Si with less than $4 \%$ misfit, making it possible to realize domain matching epitaxy [12].

In this paper, we report the fabrication and characterization of the first TiN nanobridge JJs, as well as nanoscale SQUIDs (nanoSQUIDs) based on these JJs. We observed that the superconducting transition temperature of TiN films grown on Si was higher than that of TiN films grown under the same conditions on $\mathrm{MgO}$ or sapphire. In combination with other practical advantages, such as availability and price, ease of nanomachining and low noise, this determined our choice to study TiN films and devices prepared on Si substrates. The large coherence length $\xi$ of $100 \mathrm{~nm}$ at $4.2 \mathrm{~K}$ in the TiN films allows relatively easy patterning of variable thickness nanobridge JJs that have length $\sim 100 \mathrm{~nm}, J_{c} \sim 10 \mathrm{MA} / \mathrm{cm}^{2}$ and non-hysteretic $I(V)$ characteristics at $4.2 \mathrm{~K}$. NanoSQUIDs based on variable thickness nanobridge JJs have relatively low kinetic inductance of their loops and up to $\sim 17 \mu \mathrm{V}$ peak-to-peak modulations of their $V(B)$ characteristics at $4.2 \mathrm{~K}$. Furthermore, the corrosion resistance of TiN provides long-term stability of nanoSQUIDs and other superconducting circuits based on TiN nanobridge JJs. 


\section{Materials and Methods}

TiN films were deposited by pulsed reactive DC magnetron sputtering from $50 \mathrm{~mm}$ TiN targets, primarily on $10 \mathrm{~mm} \times 10 \mathrm{~mm} \times 0.4 \mathrm{~mm} \mathrm{Si}(001)$ substrates. The edges of the substrates were oriented along the (110) and (1110) crystallographic planes of $\mathrm{Si} . \mathrm{MgO}$ and sapphire substrates were also tested for comparison. The substrates were cleaned in an ultrasonic bath successively in acetone, propanol and double-distillated deionized water. The native oxide was removed from the Si substrate by rinsing in a $10 \% \mathrm{HF}$ solution for $20 \mathrm{~s}$, followed by cleaning in double-distillated deionized water and drying by blowing with $99.9995 \%$ pure $\mathrm{N}_{2}$. The substrates were placed on a heater plate in the sputtering system, which was pumped to a base pressure of $4 \times 10^{-8}$ mbar. The TiN films were sputtered at a rate of $\sim 1 \mathrm{~nm} / \mathrm{min}$ in a gas mixture $\operatorname{Ar}(80 \%)-\mathrm{N}_{2}(20 \%)$ at a total pressure of $10^{-2}$ mbar, a DC voltage bias of $200 \mathrm{~V}$, a DC current of $100 \mathrm{~mA}$ and a heater temperature of $920^{\circ} \mathrm{C}$, which corresponded to a substrate temperature $T_{\text {sub }}$ of $\sim 800^{\circ} \mathrm{C}$. Before deposition, the chamber was outgassed at the deposition conditions for more than $1 \mathrm{~h}$ without plasma and for 10 min of pre-sputtering on the shutter in its "CLOSED" position between the target and the substrate. The gases that were used for sputtering TiN were of the best available purity: $99.9999 \%$ pure $\mathrm{Ar}$ and $99.9999 \%$ pure $\mathrm{N}_{2}$, each containing below $0.01 \mathrm{ppm}$ of oxygen, corresponding to a partial pressure of oxygen in the sputtering plasma of below $10^{-10}$ mbar.

The microstructural properties and crystallographic orientations of the TiN films were studied using high-resolution scanning electron microscopy (HRSEM), high-resolution transmission electron microscopy (HRTEM) and X-ray diffraction (XRD) in Forschungszentrum Jülich (FZJ) (Jülich, Germany). HRTEM images were recorded using a Tecnai F20 TEM at an accelerating voltage of $200 \mathrm{kV} .2 \Theta X R D$ scan patterns were acquired using a BRUKER D8 DISCOVER high resolution X-ray diffractometer with an in-plane rotation angle of $\varphi=45^{\circ}$. The dependences of the resistance of the TiN films on temperature $R(T)$ and magnetic field $R(B)$ were measured using a Quantum Design Physical Property Measurement System (PPMS). Voltage modulations of the nanoSQUIDs with magnetic field $V(B)$ at different current biases and temperatures were measured using the same PPMS. The $R(T)$ characteristics of the nanobridges and $I(V, B)$ characteristics of nanoSQUIDs were measured using a Quantum Design DynaCool system in FZJ. The dependence of the induced magnetic moment of the superconducting TiN films on magnetic field was measured using a Quantum Design Magnetic Property Measurement System (MPMS) at 1.9 K, which is the lowest stabilized temperature in this system.

Patterning of the TiN films with a spatial resolution down to $10 \mathrm{~nm}$ was performed by RIE using masks of hydrogen silsesquioxane (HSQ) electron beam resist. The $\sim 60-\mathrm{nm}$-thick $3 \%$ HSQ resist was exposed using an electron beam with an accelerating voltage of $100 \mathrm{kV}$, a current of $10 \mathrm{nA}$ and a dose of $2.5 \mathrm{mC} / \mathrm{cm}^{2}$. The design of the nanoSQUID included a third electrode, which was used for direct injection of flux bias [2,4]. Submicrometer structures were written in the resist using a 2-nm-diameter electron beam, while wider structures of current lines were written using a $10 \mathrm{~nm}$ electron beam. Contact pads were etched through Pt thin film masks that were deposited through a Ti shadow mask. A post-exposure bake was performed for $2 \mathrm{~min}$ at $120^{\circ} \mathrm{C}$. The resist was developed in $25 \%$ tetramethylammonium hydroxide (TMAH) for $20 \mathrm{~s}$ in an ultrasonic bath, before rinsing the sample in distillated and deionized water in an ultrasonic bath for $1.5 \mathrm{~min}$ following by rinsing in deionized water for $1 \mathrm{~min}$. RIE of the TiN films was performed in the clean room of the Helmholtz Nano Facility (HNF) in FZJ [13] using an Oxford Instruments PlasmalabSystem 100 with pure $\mathrm{SF}_{6}$ gas and only $25 \mathrm{~W}$ of radio frequency $(\mathrm{RF})$ plasma, which provided a selectivity of $\sim 10$ for etching the TiN films, as compared to the etch rate of the HSQ resist. Inductively coupled plasma was not used during RIE because this would reduce selectivity.

More than $100 \mathrm{TiN}$ thin film samples were produced and investigated during the last two years of optimization of the deposition parameters and nanostructuring technology. Approximately half of them were produced at the current deposition conditions and only their dimensions and the shapes of the nanobridges were adjusted. The superconducting 
transition temperatures of TiN films of similar thicknesses had a spread that was within $1 \%$. The spread of critical currents of the nanobridges was correlated with the spread in their dimensions of approximately $20 \%$ due to the sensitivity of the reactive ion etching rate to the surface cleanliness of the TiN films and the preconditioning of the RIE machine.

\section{Results}

The lowest room temperature resistivity of $\sim 15 \mu \Omega \cdot \mathrm{cm}$, the largest residual resistance ratio $R R R=R_{300 K} / R_{10 K} \cong 4$ and the largest superconducting transition temperature $T_{c} \cong 5.25 \mathrm{~K}$ were measured for 700 -nm-thick TiN films grown on $\mathrm{Si}$ substrates (see Figure 1). A similar room temperature resistivity was measured in [12] for epitaxial TiN films whose stoichiometry was confirmed using Rutherford backscattering spectrometry.

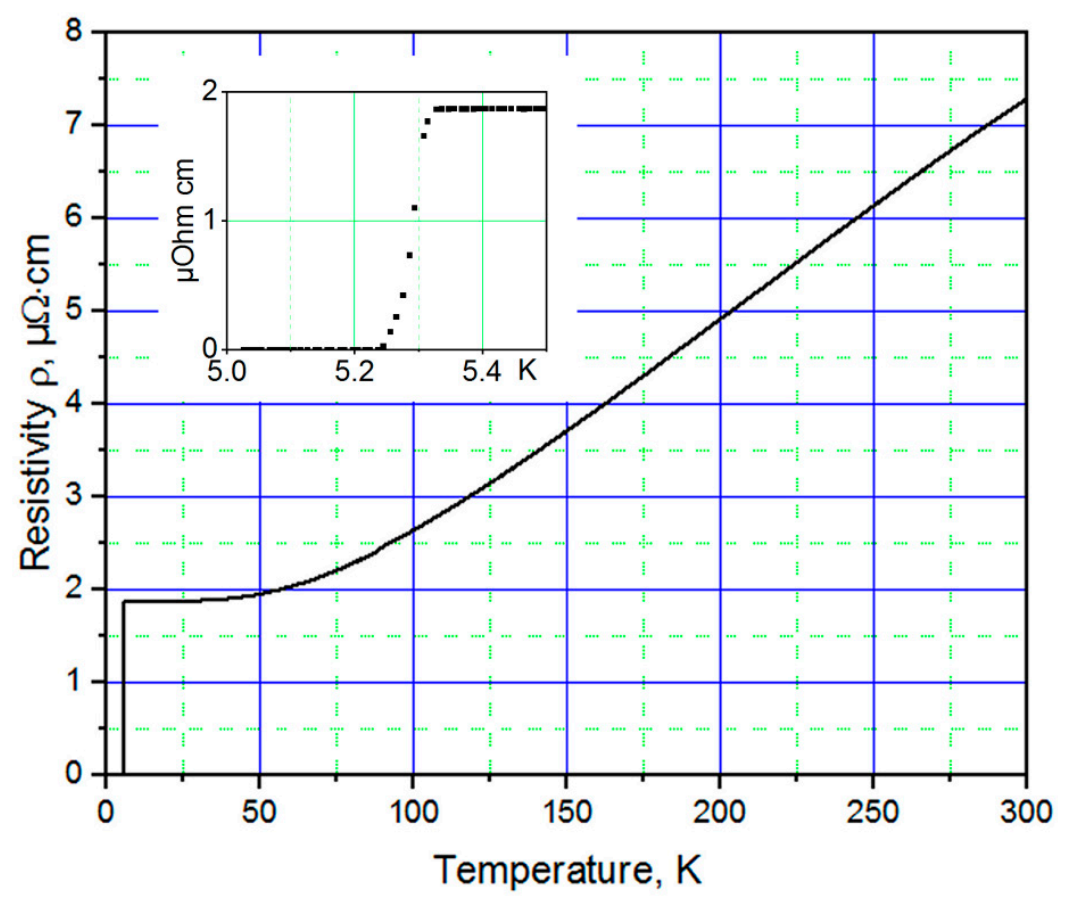

Figure 1. $R(T)$ dependence of a 700-nm-thick titanium nitride (TiN) film grown on a Si (100) substrate. The inset shows $R(T)$ dependence at the superconducting transition.

This value of $T_{c}$ is higher than that of TiN films grown under the same conditions on $\mathrm{MgO}(4.65 \mathrm{~K})$ or sapphire $(3.7 \mathrm{~K})$. Moreover, the value of $T_{c}$ for the TiN films was lower when using pristine $\mathrm{Si}$ substrates with native oxide on their surfaces $(4.8 \mathrm{~K})$ that had not been removed using $10 \%$ HF during substrate preparation. TiN films that were deposited on Si covered by a 20-nm-thick SiN buffer layer demonstrated sufficiently high values of $T_{c} \cong 5.1 \mathrm{~K}$.

The superconducting transition temperature $T_{c}$ of the TiN films depends strongly on deposition conditions and film thickness. The value of $T_{\mathcal{C}}$ is higher when using a lower base pressure and a higher substrate temperature $T_{\text {sub }}$. A reduction in film thickness to below $\sim 150 \mathrm{~nm}$ leads to an exponentially decreasing $T_{c}$ : 100-nm-thick TiN films deposited on oxide-free $\mathrm{Si}(100)$ substrates have $T_{\mathcal{C}} \cong 4.86 \mathrm{~K}$ and a residual resistance ratio RRR of $\sim 2$.

Figure 2 shows a cross-sectional HRTEM image of the interface between a Si substrate and a TiN film viewed along the [110] crystallographic direction of Si. The angle between (100) planes in the TiN film and the surface of the $\mathrm{Si}(100)$ substrate of approximately $70^{\circ}$ is consistent with a (111) orientation of the TiN film. On a larger scale, the TiN film shows a polycrystalline structure with a typical crystallite size of $5-50 \mathrm{~nm}$. The crystallite size increases with film thickness. 


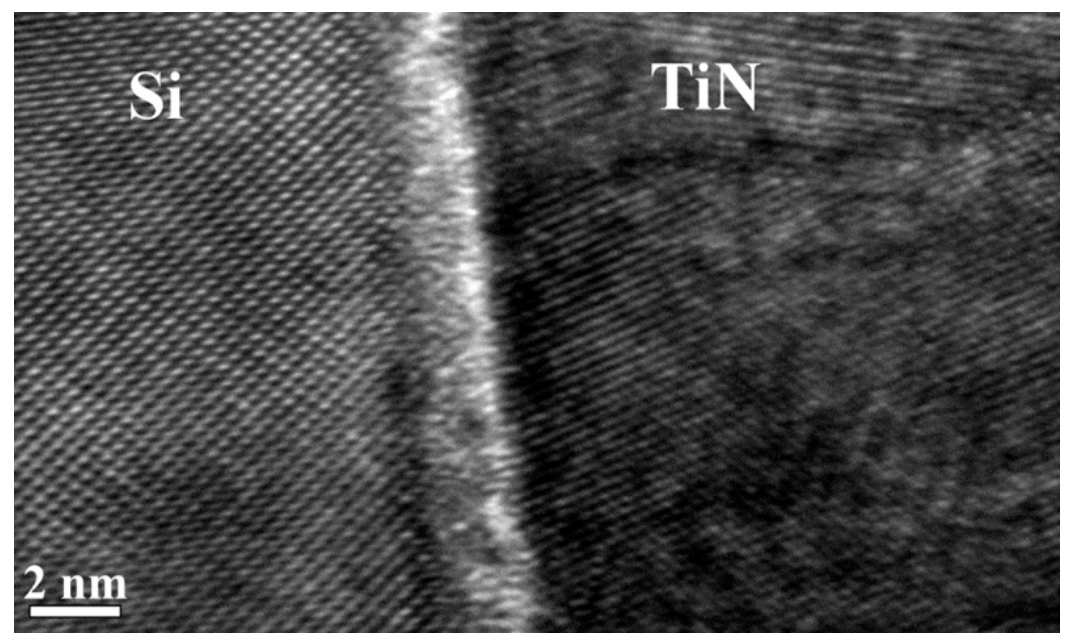

Figure 2. High-resolution TEM image of a Si (110) cross-section, showing (100) planes in the TiN film and (111) planes Si substrate, with the interface between them oriented parallel to the electron beam.

The (100) planes of the (111) oriented TiN film cross the (100)-oriented surface of the $\mathrm{Si}$ substrate. Four unit cells along the $(100)$ planes of the TiN film $(4 \times 0.424 \mathrm{~nm})$ match three unit cells of the (100)-oriented $\mathrm{Si}$ substrate $(3 \times 0.543 \mathrm{~nm})$ with a $4 \%$ misfit. This can provide a weak coupling between the TiN film and Si surface. Similar misfits have been reported for epitaxial (200)-oriented TiN films that were prepared using pulsed laser deposition [12].

The (111) orientation is predominant for TiN films that have a thickness of below $200 \mathrm{~nm}$, while thicker films also contain a (200) orientation contribution. Figure 3 shows an XRD scan recorded from a 400-nm-thick TiN film grown on an oxide-free Si substrate, containing peaks from both (111) and (200) orientations. A smaller ratio between the amplitudes of the (200) and (111) peaks and a smaller value of $T_{\mathcal{C}}$ were observed for thinner TiN films and/or lower deposition temperatures $T_{\text {sub }}$. The amplitude of the (200) TiN peak increased relative to the (111) peak with increasing film thickness.

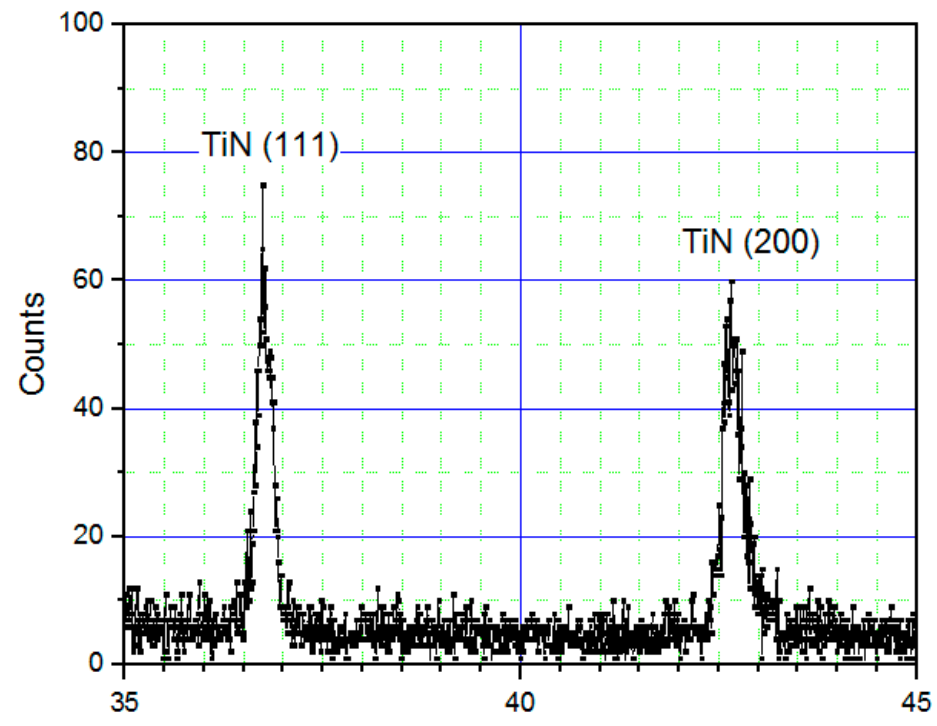

Angle $2 \Theta$, deg.

Figure 3. XRD scan recorded from a 400-nm-thick TiN film deposited on an oxide-free Si substrate. 
Figure 4 shows the magnetoresistance $R(B)$ of a 100-nm-thick TiN film recorded using PPMS with the magnetic field applied perpendicular to the film surface at $1.8 \mathrm{~K}$. The upper critical magnetic field $\mathrm{B}_{\mathrm{c} 2}$ is approximately $0.21 \mathrm{~T}$, which corresponds to a superconducting coherence length $\xi(1.8 \mathrm{~K})$ of $\sqrt{\Phi_{0} / 2 \pi B_{C 2}} \cong 39 \mathrm{~nm}$. This value is significantly larger than the value of $\xi_{\mathrm{Nb}}$ of $\sim 10 \mathrm{~nm}$ measured for $\mathrm{Nb}$ thin films, which is beneficial for the fabrication of JJs. Moreover, at $4.2 \mathrm{~K}$ the coherence length increased to $\sim 107 \mathrm{~nm}$ as a result of the temperature dependence $\xi \propto\left(1-\frac{T}{T_{c}}\right)^{-\frac{1}{2}}$. The irreversibility magnetic field $B_{\text {irr }} \cong 0.12$ $\mathrm{T}$ corresponds to the threshold magnetic field for creep of Abrikosov vortices, which is therefore the upper limit of the magnetic field for low noise operation of Josephson devices.

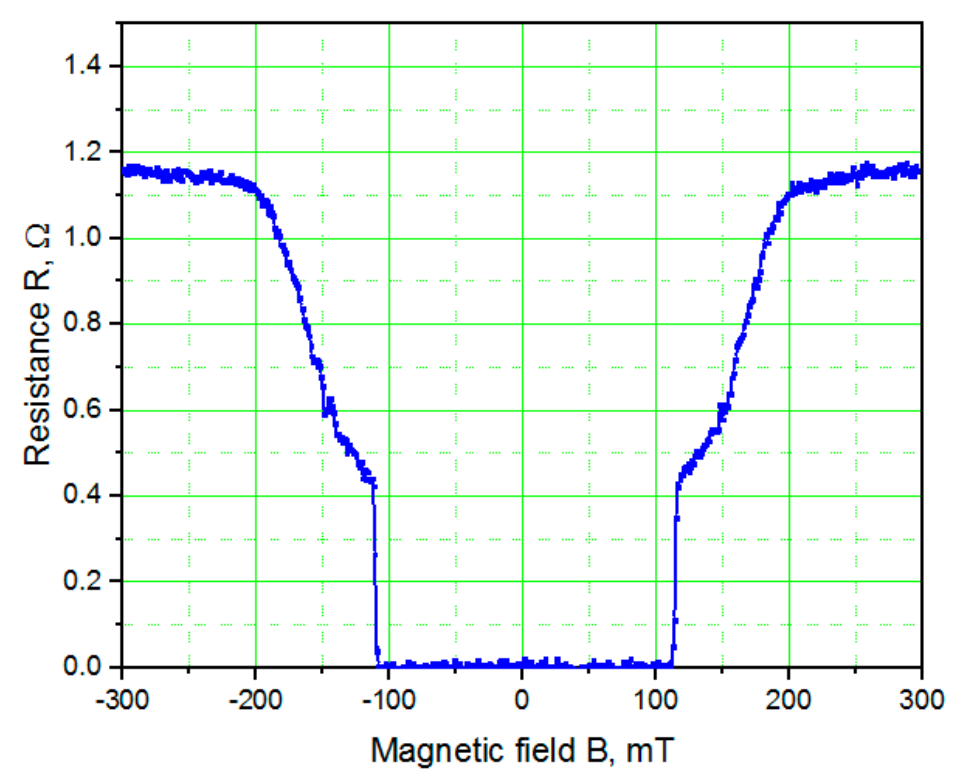

Figure 4. Magnetoresistance $R(B)$ of a $100-\mathrm{nm}$-thick TiN film recorded at $1.8 \mathrm{~K}$. The magnetic field was oriented perpendicular to the surface of the film.

Figure 5 shows the dependence of magnetic moment on magnetic field $M(B)$ for a 1.2- $\mu \mathrm{m}$-thick TiN film that had been deposited on a Si substrate with a 20-nm-thick SiN buffer layer measured using MPMS at $1.9 \mathrm{~K}$. The magnetic field was applied parallel to the film surface. For such a thicker TiN film, the irreversibility magnetic field $B_{i r r}$ of $\sim 50 \mathrm{mT}$ is lower, as a result of weaker pinning of Abrikosov vortices in films that have better crystallinity. The upper critical magnetic field $B_{c 2}$ of $\sim 0.15 \mathrm{~T}$ corresponds to a coherence length $\xi(1.9 \mathrm{~K})$ of $\sim 50 \mathrm{~nm}$. At $4.2 \mathrm{~K}$, the coherence length increased as a result of the temperature dependence $\xi \propto\left(1-\frac{T}{T_{c}}\right)^{-\frac{1}{2}}$ to $\sim 105 \mathrm{~nm}$, which is approximately the same as that measured for thinner films because the larger $\xi(1.9 \mathrm{~K})$ is compensated at $4.2 \mathrm{~K}$ by the higher value of $T_{c}$. 


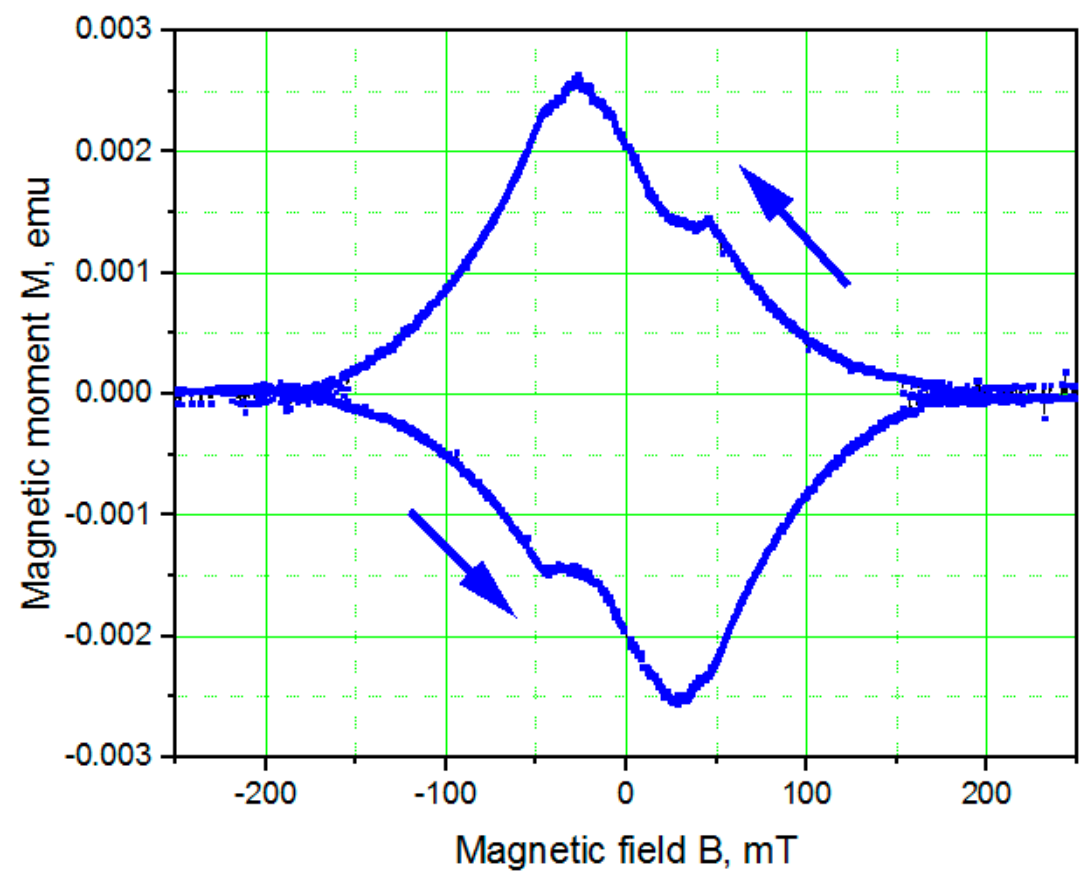

Figure 5. Dependence of magnetic moment $M(B)$ of a 1.2- $\mu$ m-thick TiN film deposited on a Si substrate with a $20-\mathrm{nm}$-thick SiN buffer layer recorded at $1.9 \mathrm{~K}$. The magnetic field was applied parallel to the surface of the film.

Nanobridge JJs were fabricated from 100-nm-thick TiN films using 30-nm-wide bowtie-type bridges in the mask of the HSQ resist. During RIE, isotropic etching with a $50 \mathrm{~nm}$ undercut was observed, as shown in Figure 6.

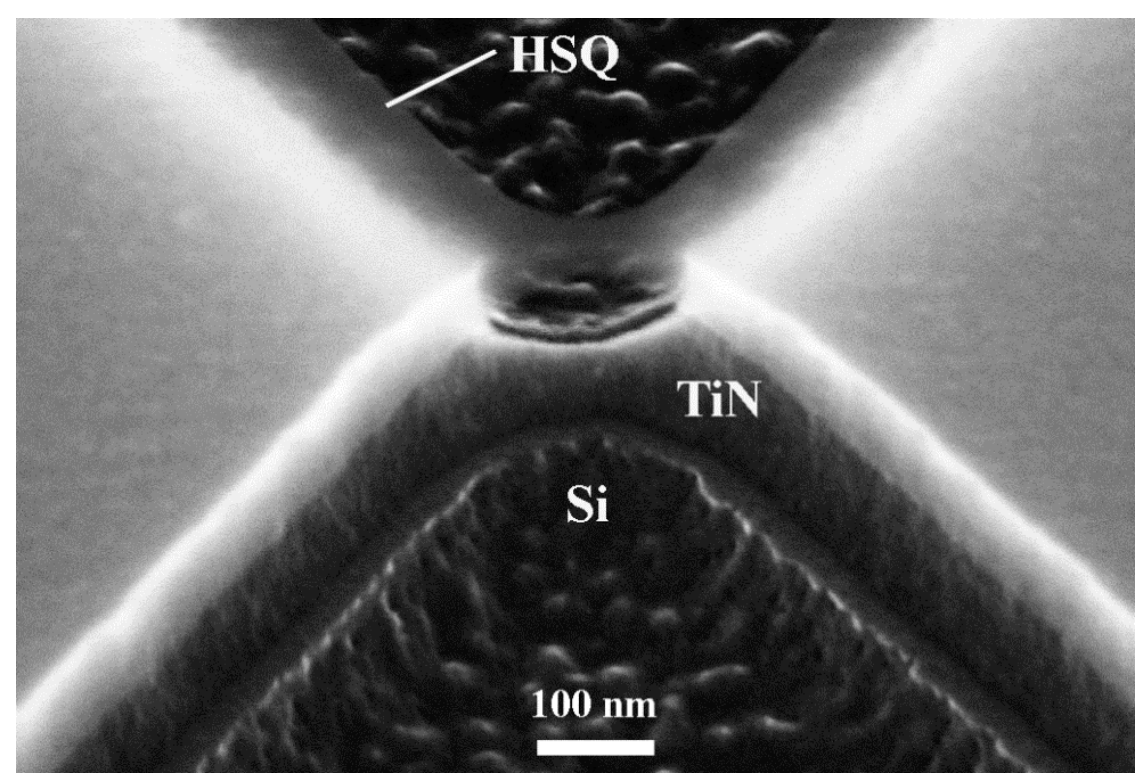

Figure 6. High-resolution SEM image of a TiN nanobridge Josephson junction (JJ) recorded at a $45^{\circ}$ sample tilt angle.

As a result of the undercut, the JJ takes the form of a variable thickness nanobridge that is much thinner and narrower in its middle part. The cross-section is a triangle, with an average width of $\sim 20 \mathrm{~nm}$ that is much smaller than the coherence length in the TiN film $\xi(4.2 \mathrm{~K}) \cong 105 \mathrm{~nm}$. After overcoming a small threshold, likely due to the presence of a thin $\mathrm{SiN}$ layer that grew naturally during presputtering at the interface between $\mathrm{Si}$ and TiN, the RIE continued into the Si substrate. 
Figure 7 shows an HRSEM image of a TiN nanoSQUID. The second superconducting current lead of one of the electrodes can be used for the direct injection of magnetic flux bias into the SQUID loop [2,4].

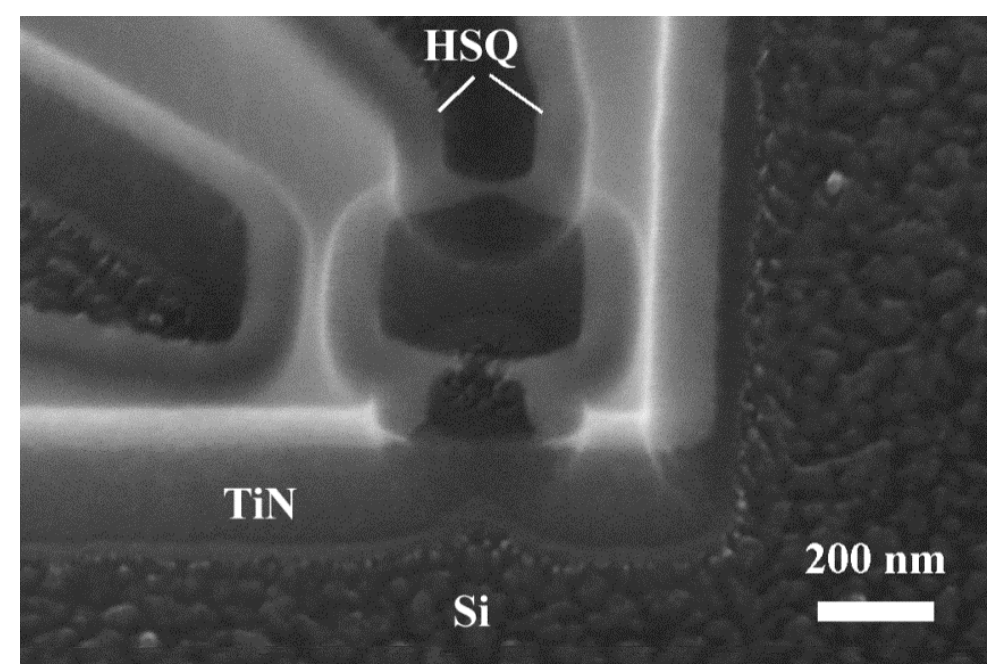

Figure 7. High-resolution SEM image of a TiN nanometer-scale superconducting quantum interference device (nanoSQUID) recorded at a $45^{\circ}$ sample tilt angle.

Figure 8 shows the $I(V)$ characteristics of a TiN nanoSQUID measured in magnetic fields of 0 and $4 \mathrm{mT}$ at $4.2 \mathrm{~K}$. At zero magnetic field, this nanoSQUID has a non-hysteretic $I(V)$ characteristic at $I_{c} \cong 50 \mu \mathrm{A}$. NanoSQUIDs with thicker JJs have larger critical currents, but their $I(V)$ curves remain non-hysteretic up to $I_{c}>100 \mu \mathrm{A}$, which is much larger than the maximal critical current of a non-hysteretic $\mathrm{Nb}$ nanoSQUID based on nanobridge JJs [14]. The observed large amplitude of modulation is related to the relatively low kinetic inductance of a nanoSQUID with variable thickness nanobridge JJs.

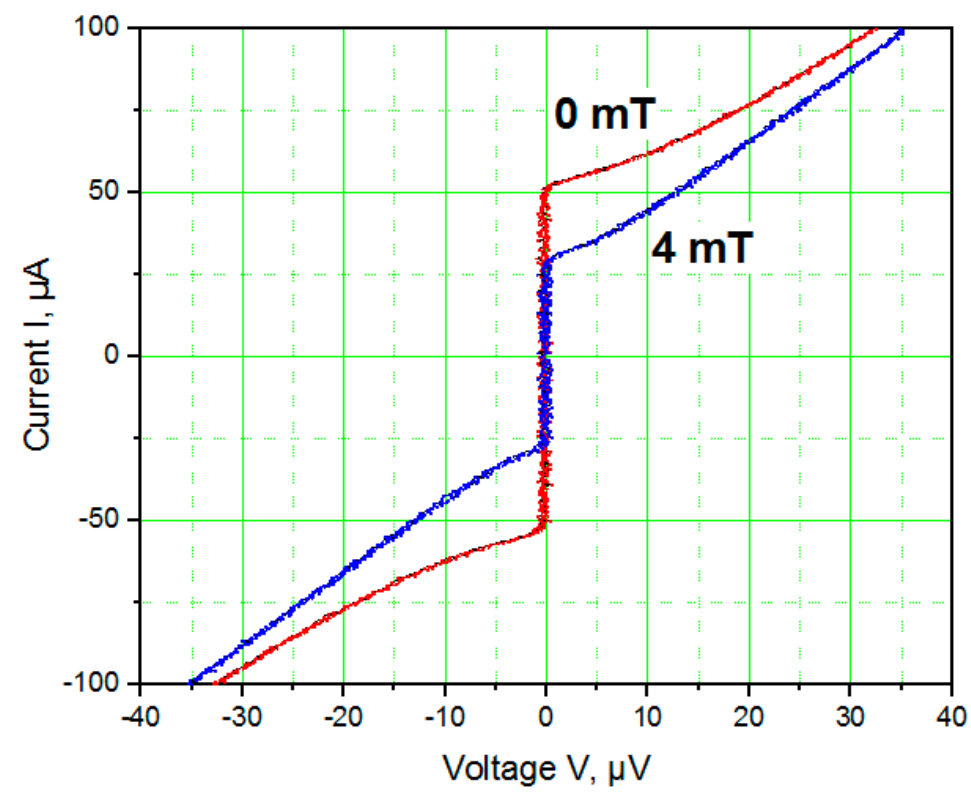

Figure 8. $I(V)$ characteristics of a TiN nanoSQUID measured in magnetic fields of $0 \mathrm{mT}$ and $4 \mathrm{mT}$ at $4.2 \mathrm{~K}$.

Figure 9 shows the $V(B)$ dependence of the TiN nanoSQUID measured for a bias current of $60 \mu \mathrm{A}$ at $4.2 \mathrm{~K}$. For this design of nanoSQUID, the effective area and period of critical current modulation on the magnetic field are $0.36 \mu \mathrm{m}^{2}$ and $6 \mathrm{mT}$, respectively, in 
accordance with earlier observations for $\mathrm{Nb}$ nanoSQUIDs [2,4]. The $V(B)$ dependence of the TiN nanoSQUID is not typical for SQUIDs and looks more like the Fraunhofer diffraction pattern that is typical for the magnetic field dependence of the critical current of a single Josephson junction. This can be a consequence of the three-angle cross-sectional shapes of the nanobridge JJs. The effective area of the nanoSQUID is not exactly defined and there is an interference of different passes for Cooper pairs, which can mimic the diffraction pattern of a slit in optics (Fraunhofer pattern) and in an individual JJ.

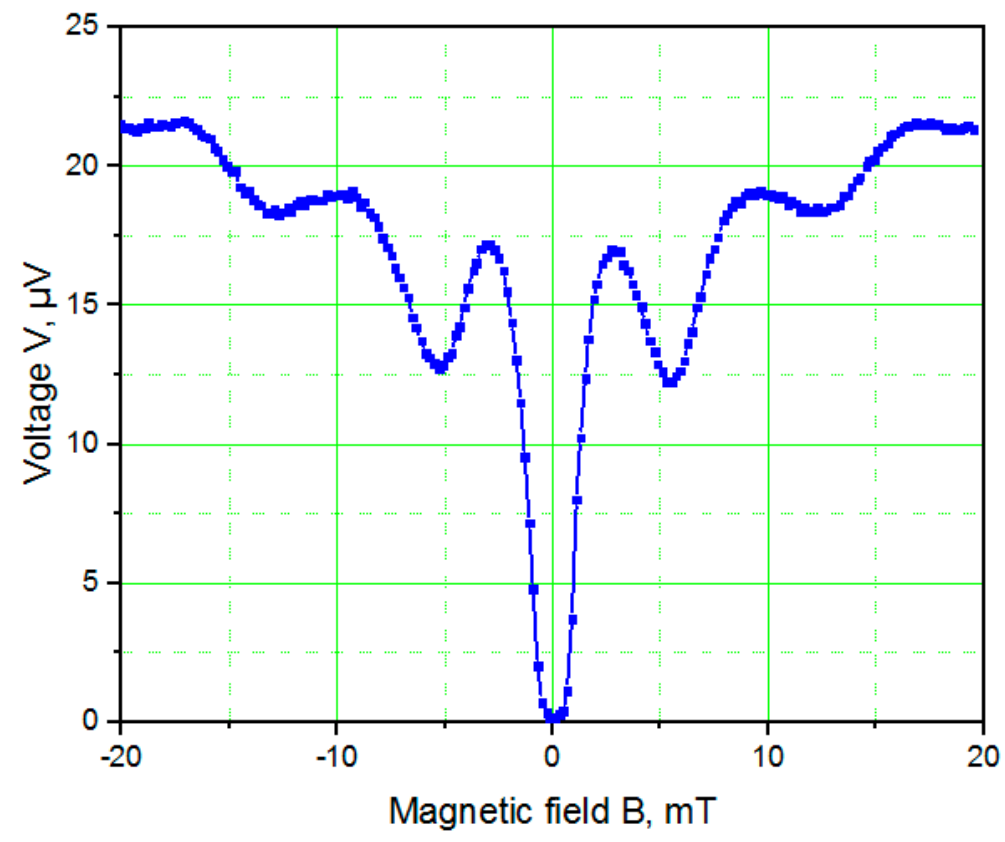

Figure 9. $V(B)$ dependence of the TiN nanoSQUID measured at $4.2 \mathrm{~K}$.

\section{Discussion}

This is the first time that TiN has been used for the preparation of nanobridge JJs and SQUIDs. It has several properties that are advantageous for superconducting nanobridge electronics: (a) a large coherence length in thin films; (b) a superconducting transition temperature $T_{c}$ of $\sim 5 \mathrm{~K}$, which is appropriate for nanobridge operation at $4.2 \mathrm{~K}$; (c) technological compatibility with $\mathrm{Si}$; (d) long-term stability due to superior oxidation resistance and thermal and chemical stability; (e) mechanical hardness and stability; (f) low microwave losses; (g) availability and relatively low cost; (h) highly selective RIE; (i) it is a plasmonic material with tunable optical properties; (j) it has other properties that are advantageous for applications in the microelectronics industry [15].

The superconducting transition temperature $T_{c}$ of the TiN films depends strongly on the choice of substrate material, the deposition conditions and the film thickness. Surprisingly, TiN films have the highest values of $T_{\mathcal{C}}$ when they are grown on pure $\mathrm{Si}$ (100) substrates, despite the $28 \%$ lattice misfit and polycrystalline microstructure of the TiN films on these substrates. This behavior may result from the minimal diffusion of oxygen from the substrate into the TiN film for a pure Si substrate. The unintended incorporation of oxygen in a TiN film is the strongest factor in suppressing superconducting properties in TiN films [16]. The use of a sufficiently low base pressure of $<10^{-7} \mathrm{mbar}$ in the deposition chamber and outgassing of the chamber at the deposition temperatures helps to reduce deleterious oxygen outgassing during film deposition. A substrate temperature $T_{\text {sub }}$ of $\sim 800{ }^{\circ} \mathrm{C}$ was found to be optimal to achieve the highest value of $T_{\mathcal{C}}$, as well as a golden color of the 100-nm-thick TiN films.

The TiN films are polycrystalline with two preferred orientations: (111) and (200). HRTEM images show primarily a (111) orientation of the film near its interface with the Si substrate. XRD also shows that TiN films that are thinner than $\sim 200 \mathrm{~nm}$ have a 
predominantly (111) orientation. XRD scans of thicker films show peaks corresponding to a (200) orientation. Films with (111) orientations have the lowest internal stress energy, while the (200) plane has the lowest surface energy $[17,18]$. The polycrystalline microstructure of the TiN films may result from the accommodation of internal stress at the interface with the substrate as a consequence of the $28 \%$ lattice misfit. An increase in film thickness reduces the contribution of interface stress energy far from the interface relative to the surface energy, leading to an increase in the proportion of (200)-oriented grains.

TiN films have the largest coherence length $\xi(4.2 \mathrm{~K})$ of $\sim 105 \mathrm{~nm}$ when compared to films of other materials that are superconducting at $4.2 \mathrm{~K}$. As a result of the large coherence length, the effect of grain boundaries on the critical current of a TiN film is much smaller than in $\mathrm{Nb}$ thin films $(\widetilde{\zeta}(4.2 \mathrm{~K}) \cong 15 \mathrm{~nm})$ or in $\mathrm{YBa}_{2} \mathrm{Cu}_{3} \mathrm{O}_{7-\mathrm{x}}(\xi(4.2 \mathrm{~K}) \cong 1.2 \mathrm{~nm})$. The polycrystalline microstructure of TiN films does not degrade their superconducting properties significantly but is beneficial for isotropic RIE during the preparation of JJs.

As a result of the large coherence length in TiN thin films, it was found that the preparation of nanobridge JJs that are non-hysteretic at $4.2 \mathrm{~K}$ was much easier than for nanobridge JJs based on other superconducting materials. The spatial resolution of electron beam resists is sufficient for the preparation of nanobridges, in which all three dimensions (length, thickness and width) are equal to or smaller than the superconducting coherence length in this material, thereby fulfilling the conditions for "ideal" JJs that were formulated by K. K. Likharev [1]. Moreover, by using an undercut effect during RIE, it is possible to realize variable thickness nanobridge JJs, whose thickness is much smaller in the middle of the nanobridge when compared to the thickness of the same film in the nearby area of the electrodes. This is an important boundary condition for the theoretical description of nanobridge JJs [19-21]. Thick superconducting electrodes are also a precondition for low kinetic inductance of the SQUID loop, which is important for relatively large critical current modulation $I_{c}(B)$ and high resolution of the SQUIDs.

The much higher critical current density in the nanobridge JJs is associated with the different physical nature of the transport of Cooper pairs, when compared to the dominant contribution of the tunneling current-transport mechanism in the supercurrent in superconductor-insulator-superconductor (SIS) tunnel JJs (see [1], [22], etc.). The GinzburgLandau (GL) equation, which is appropriate for nanobridge JJs, can be written in a onedimensional approximation in the form ([22-24])

$$
\xi^{2} \frac{d^{2} f}{d x^{2}}+f-f^{3}=0
$$

where $\xi$ is the coherence length and $f=\frac{\Psi}{\Psi_{\infty}}$ is the normalized order parameter. In the case of a short length of nanobridge $L<<\xi$, the first term dominates and the equation is reduced to the Laplace equation $d^{2} f / d x^{2}=0$, for which the solution is the linear function $f=a+b x$, with $x$ ranging from 0 to $L$

$$
f=\left(1-\frac{x}{L}\right)+\left(\frac{x}{L}\right) e^{i \Delta \varphi}
$$

where $\Delta \varphi$ is the phase difference between the wave functions in the electrodes. Insertion of the latter equation for $f$ into the GL equation for the superconducting current $I_{S}$ results in the Josephson current-phase dependence $I_{S}=I_{C} \sin (\Delta \varphi)$.

The energy sensitivity of the nanoSQUID at the operating temperature of $4.2 \mathrm{~K}$ is limited to a first approximation by Johnson noise, according to the expression [25].

$$
\frac{S_{\Phi}}{2 L}=\frac{S_{\mathrm{V}}}{\left(\frac{\partial V}{\partial \Phi}\right)^{2} 2 L_{k}}=\frac{16 k_{B} T R_{N}}{\left(\frac{\partial V}{\partial \Phi}\right)^{2} 2 L_{k}} \cong 12 \hbar
$$

where the values $R_{N}=0.08 \Omega$ and $\partial V / \partial \Phi \cong 126 \mu \mathrm{V} / \Phi_{0}$ were measured from Figure 9 . The relatively low intrinsic energy noise of $\sim 12 \hbar$ results from the very low resistance $R_{N}$. The kinetic inductance of a nanoSQUID loop with variable-thickness nanobridge JJs $L_{k}$ is approximately $8 \mathrm{pH}$. We estimate the nanoSQUID to have a flux sensitivity $S_{\Phi}{ }^{1 / 2}$ 
$\cong 68 \mathrm{n} \Phi_{0} / \mathrm{Hz}$, a magnetic field resolution $B_{n}=(\partial B / \partial \Phi) S_{\Phi}{ }^{1 / 2} \cong 0.4 \mathrm{nT} / \sqrt{ } \mathrm{Hz}$ and a spin sensitivity $S_{n}{ }^{1 / 2}=S_{\Phi}{ }^{1 / 2} r / r_{e} \cong 11 \mu_{\mathrm{B}} / \sqrt{ } \mathrm{Hz}$, where $r_{e}=2.82 \times 10^{-15} \mathrm{~m}$ is the classical electron radius [26].

The influence of quantum fluctuations on the operation of TiN nanoSQUIDs at $4.2 \mathrm{~K}$ can be neglected. However, it cannot be excluded in the case of their potential applications at temperatures below $100 \mathrm{mK}$. The large coherence length $\xi(0)$ of the TiN films allows the realization of non-hysteretic variable-thickness TiN nanobridge JJs in the milliKelvin temperature range, as well as their combination with high quality resonators made from the same material.

\section{Conclusions}

The observed large coherence length $\xi(4.2 \mathrm{~K}) \sim 105 \mathrm{~nm}$ in TiN thin films allows relatively easy patterning of variable thickness nanobridge JJs with length $\sim 100 \mathrm{~nm}$, $J_{c} \sim 10 \mathrm{MA} / \mathrm{cm}^{2}$ and non-hysteretic $I(V)$ characteristics at $4.2 \mathrm{~K}$. NanoSQUIDs based on variable thickness nanobridge JJs demonstrate relatively low kinetic inductance of the loops and up to $\sim 17 \mu \mathrm{V}$ peak-to-peak modulation of the $V(B)$ characteristics at $4.2 \mathrm{~K}$. The high corrosion resistance of TiN provides long term stability of nanoSQUIDs and other superconducting circuits that are based on TiN nanobridge JJs. The technology offers prospects for superconducting nanobridge electronics and quantum computing.

Author Contributions: Conceptualization, methodology and original draft preparation, M.I.F.; investigation, formal analysis, data curation, M.I.F. and Y.L.; supervision and resources, M.I.F. and R.E.D.-B. All authors contributed to the review and editing of the manuscript. All authors have read and agreed to the published version of the manuscript.

Funding: This research received no external funding.

Acknowledgments: The authors gratefully acknowledge the possibility to perform parts of the work at ER-C-1, HNF, PGI-4/JCNS-2, PGI-5 and PGI-7 in Forschungszentrum Jülich GmbH. The authors also gratefully acknowledge S. Aussen and S. Hoffmann-Eifert for the XRD measurements, V. S. Stolyarov, A. A. Golubov and H. Fiadziushkin for fruitful discussions and H. Stumpf, M. Nonn, S. Trellenkamp, F. Lentz, O. Petracic, S. Nandi, B. Schmitz and R. Borowski for technical assistance.

Conflicts of Interest: The authors declare no conflict of interest.

\section{References}

1. Likharev, K.K. Superconducting weak links. Rev. Mod. Phys. 1979, 51, 101-159. [CrossRef]

2. Rodrigo, R.; Faley, M.; Dunin-Borkowski, R. NanoSQUIDs based on Nb nanobridges. J. Phys. Conf. Ser. 2020, $1559,012011$. [CrossRef]

3. Shishkin, A.; Skryabina, O.V.; Gurtovoi, V.; Dizhur, S.; Faley, M.; Golubov, A.A.; Stolyarov, V.S. Planar MoRe-based direct current nanoSQUID. Supercond. Sci. Technol. 2020, 33, 065005. [CrossRef]

4. Faley, M.; Bikulov, T.; Bosboom, V.; Golubov, A.A.; Dunin-Borkowski, R.E. Bulk nanomachining of cantilevers with Nb nanoSQUIDs based on nanobridge Josephson junctions. Supercond. Sci. Technol. 2021, 34, 035014. [CrossRef]

5. Shelly, C.D.; See, P.; Ireland, J.; Romans, E.J.; Williams, J.M. Weak link nanobridges as single flux quantum elements. Supercond. Sci. Technol. 2017, 30, 095013. [CrossRef]

6. Vershinin, N.; Filonov, K.; Straumal, B.; Gust, W.; Wiener, I.; Rabkin, E.; Kazakevich, A. Corrosion behaviour of the protective and decorative TiN coatings on large area steel strips. Surf. Coat. Technol. 2000, 125, 229-232. [CrossRef]

7. Vissers, M.R.; Gao, J.; Wisbey, D.S.; Hite, D.A.; Tsuei, C.C.; Corcoles, A.D.; Steffen, M.; Pappas, D.P. Low loss superconducting titanium nitride coplanar waveguide resonators. Appl. Phys. Lett. 2010, 97, 232509. [CrossRef]

8. Sage, J.M.; Bolkhovsky, V.; Oliver, W.D.; Turek, B.; Welander, P.B. Study of loss in superconducting coplanar waveguide resonators. J. Appl. Phys. 2011, 109, 063915. [CrossRef]

9. Chang, J.B.; Vissers, M.R.; Córcoles, A.D.; Sandberg, M.; Gao, J.; Abraham, D.W.; Chow, J.M.; Gambetta, J.M.; Rothwell, M.B.; Keefe, G.A.; et al. Improved superconducting qubit coherence using titanium nitride. Appl. Phys. Lett. 2013, $103,012602$. [CrossRef]

10. Wenner, J.; Barends, R.; Bialczak, R.C.; Chen, Y.; Kelly, J.; Lucero, E.; Mariantoni, M.; Megrant, A.; O’Malley, P.J.J.; Sank, D.; et al. Surface loss simulations of superconducting coplanar waveguide resonators. Appl. Phys. Lett. 2011, 99, 113513. [CrossRef]

11. Makise, K.; Sun, R.; Terai, H.; Wang, Z. Fabrication and Characterization of Epitaxial TiN-Based Josephson Junctions for Superconducting Circuit Applications. IEEE Trans. Appl. Supercond. 2014, 25, 1-4. [CrossRef] 
12. Narayan, J.; Tiwari, P.; Chen, X.; Singh, J.; Chowdhury, R.; Zheleva, T. Epitaxial growth of TiN films on (100) silicon substrates by laser physical vapor deposition. Appl. Phys. Lett. 1992, 61, 1290-1292. [CrossRef]

13. Helmholtz Nano Facility (HNF). Available online: https://www.fz-juelich.de/hnf//DE/Home/home_node.html (accessed on 11 February 2021).

14. Troeman, A.G.P.; van der Ploeg, S.H.W.; Il'Ichev, E.; Meyer, H.-G.; Golubov, A.A.; Kupriyanov, M.Y.; Hilgenkamp, H. Temperature dependence measurements of the supercurrent-phase relationship in niobium nanobridges. Phys. Rev. B 2008, 77, 024509 . [CrossRef]

15. Wittmer, M. Properties and microelectronic applications of thin films of refractory metal nitrides. J. Vac. Sci. Technol. A 1985, 3 , 1797-1803. [CrossRef]

16. Torgovkin, A.; Chaudhuri, S.; Ruhtinas, A.; Lahtinen, M.; Sajavaara, T.; Maasilta, I.J. High quality superconducting titanium nitride thin film growth using infrared pulsed laser deposition. Supercond. Sci. Technol. 2018, 31, 055017. [CrossRef]

17. Pelleg, J.; Zevin, L.; Lungo, S.; Croitoru, N. Reactive-sputter-deposited TiN films on glass substrates. Thin Solid Films 1991, 197, 117-128. [CrossRef]

18. Oh, U.C.; Je, J.H. Effects of strain energy on the preferred orientation of TiN thin films. J. Appl. Phys. 1993, 74, 1692-1696. [CrossRef]

19. Kupriyanov, M.Y.; Likharev, K.K.; Maslova, L.A. The Js $(\varphi)$ relationship, Abrikosov vortices and Josephson vortices in variable thickness bridges. In Proceedings of the 14th International Conference on Low Temperature Physics, Otaniemi, Finland, 14-20 August 1975.

20. Kupriyanov, M.Y.; Lukichev, B.F. Steady-state properties of a quasi-onedimensional variable-thickness bridges. Low Temp. Phys. 1981, 7, 281-287.

21. Gubankov, V.N.; Koshelets, V.P.; Ovsyannikov, G.A. Coherent effects in superconducting bridges of variable thickness. J. Exp. Theor. Phys. 1968, 71, 348-358.

22. Aslamazov, L.G.; Larkin, A.I. Josephson effect in superconducting point contacts. Pis'ma Zh. Eksp. Teor. Fiz. 1968, 9, 150-154.

23. Schmidt, V.V. The Physics of Superconductors. In The Physics of Superconductors; Springer: Berlin/Heidelberg, Germany, 1997.

24. Tinkham, M. Introduction to Superconductivity; McGraw-Hill, Inc.: New York, NY, USA, 1996; ISBN 0-07-064878-6.

25. Tesche, C.D.; Clarke, J. dc SQUID: Noise and optimization. J. Low Temp. Phys. 1977, 29, 301-331. [CrossRef]

26. Ketchen, M.; Awschalom, D.; Gallagher, W.; Kleinsasser, A.; Sandstrom, R.; Rozen, J.; Bumble, B. Design, fabrication, and performance of integrated miniature SQUID susceptometers. IEEE Trans. Magn. 1989, 25, 1212-1215. [CrossRef] 